\title{
The Effect of Early Post Hatch Feeding Times on the Growth and Development of the Gastrointestinal Tract of Mule Ducklings to Five Days of Age
}

EAuthor(s)

Williams MA' (iD https://orcid.org/0000-0001-8682-9597 Lallo CHO" (D) https://orcid.org/0000-0001-5499-8257 Sundaram VIII (iD https://orcid.org/0000-0002-7665-2836

Department of Food Production, Faculty of Food and Agriculture, The University of the West Indies St. Augustine Campus.

Open Tropical Forage and Animal-Production Laboratory, Department of Food Production Faculty of Food and Agriculture, The University of the West Indies St. Augustine Campus.

III Department of Basic Veterinary Sciences, School of veterinary medicine, Faculty of Medical Sciences, Mt.Hope, The University of the West Indies St. Augustine Campus.

\section{-Mail Address}

Corresponding author e-mail address Melissa A. Williams

Department of Food Production, Faculty of Food and Agriculture, The University of the West Indies, St.Augustine campus.

Phone: 1868-4780095

Email: akeela.williams08@gmail.com

\section{ABSTRACT}

The present study evaluated the effects of early post-hatch feeding on the growth and development of the gastrointestinal tract (GIT) of mule ducks reared in the humid tropics to five days of age. A total of 120 newly hatched mule ducklings were selected and randomly assigned to four treatment groups. Each with 30 birds, (T1, T2, T3, and T4) is based on four feeding regimes (feed accessed at 3, 24, 36, and 48 hours post-hatch) for five days. The ducklings were euthanized each day and the parameters: small intestinal samples were processed for histological analysis and histometrical parameters including villi length and width, crypt width and depth, and the number of goblet cells per villus was measured, statistically analyzed using ANOVA and tabulated. The results of this study indicated that the earliest post-hatch feeding (at 3 hours post-hatch) showed the highest body weight $(99.65 \mathrm{~g})$, heavier $(\mathrm{GIT})$ organ $(p<0.05)$, highest duodenal length $(120.71 \mathrm{~cm})$, than other feeding times. The villi height of the three segments of the GIT increased ( $p>0.05$ ) continuously regardless of the post-hatch feeding times. Based on the results, it was concluded that feeding at 3 hours post-hatch promoted the growth and development of the GIT faster than 24, 36, and 38 hours post hatch feeding.

\section{INTRODUCTION}

Globally duck farming has shown a marked increase due to economic reasons (King et al. 2000). Within the CARICOM region, particularly Trinidad, there has been an increase in the production of mule ducks. Mule ducks possess the attributes of a rapid growth rate, good breast muscle yield, lean carcass, and a lack of sexual dimorphism which makes it an ideal species for the poultry industry (Lallo \& Ramraj, 2008).

However, under normal hatchery and farm conditions feed is typically not offered to mule ducklings until approximately 32-48 hours post-hatch. This delay can result in decreased body weight and malnutrition of the yolk has insufficient nutrient content (Noy \& Sklan, 1999). Therefore, it is typical within the first 24-72 hours post-hatch that mule ducklings rely solely on the residual yolk sac for nutrients. A further delay in feed access is brought on by the delivery of ducklings to grow-out farms. This negatively impacts the overall development of the gastrointestinal tract and growth performance; thus producing a lower final body weight for mule ducklings (Dibner, 1998).

Studies have demonstrated that the immediate access of birds to feed and water increases subsequent performance in both chick and poults via mechanisms, including stimulation of yolk sac utilization and increased intestinal development (Noy \& Sklan, 1998; 2002; Sklan, 2003; Uni et al., 2003). Moreover, the development of the GIT is an important aspect of growth, especially the development of digestive functional organisms during the early post-hatching period of chicks (Nistan et al., 1991a). 
The practice of early post-hatch feeding of broiler chicks, turkey poults, and goslings increases the rate of yolk sac reabsorption and utilization by enhancing intestinal villi growth to optimize the absorptive capacity of the gastrointestinal tract at an earlier age, which in turn promotes increases in body weight of the birds up to market age. There is limited information about whether post-hatch feeding would provide similar benefits in mule ducks. However, the mule duck received much less attention because commercial poultry operations are dominated by broiler and turkeys. Therefore this study aimed to evaluate the effects of early post-hatch feeding on the growth and development of the gastrointestinal tract up to five days of mule ducks reared in the humid tropical climate of Trinidad.

\section{MATERIALS AND METHODS}

\section{Location and Climate}

Trinidad is located within the tropical region and experiences hot and humid environmental conditions with minimum and maximum temperatures of 24.1 and $36.15^{\circ} \mathrm{C}$; respectively and humidity of $80.21 \%$. There are two seasons: a dry season occurs from January to May and a rainy season from June to December. This experiment was conducted during the rainy season at The University of the West Indies Field Station Farm, Mt. Hope $\left(10^{\circ} 38^{\prime} 15^{\prime \prime}\right.$ North $61^{\circ} 25^{\prime} 39^{\prime \prime}$ West), Trinidad and Tobago.

\section{Housing and Management}

A total of one hundred and twenty (120) newly hatched mule ducklings obtained from a local hatchery were raised in an open-sided, naturally-ventilated house, in $121.9 \mathrm{~cm} \times 61 \mathrm{~cm}$ cages. They were assigned in a completely randomized design to four post-hatch feeding treatments $(T)$ with feeding at different times after hatching: T1 (3 hrs), T2 (24 hrs), T3 (36 hrs), and T4 (48 hrs). These treatments were selected based on the time taken locally for mule ducks to arrive at grow-out farms. Each treatment was replicated 6 times with 5 birds/replicate for a total of 30 birds per treatment. T1 received both water and feed 3 hours post-hatch, while the other treatments received only water until they were fed at 24, 36, and 48 hours, respectively. Feed was offered according to the National Research Council standards (NRC, 1994) and refusal was measured daily to determine feed intake; water was provided ad libitum. No vaccination was conducted in keeping with local production practices. The experimental period was conducted for five days.
Table 1 - Showing Ingredient and composition of a commercial starter feed.

\begin{tabular}{lc}
\hline Ingredients (g/kg-1 DM) & Starter \\
\hline Soyabean Meal (470g CPkg $\left.{ }^{1} \mathrm{DM}\right)$ & 415.2 \\
Ground corn & 356.4 \\
\hline Rice Bran & 80.0 \\
\hline Broken Rice & 60.6 \\
\hline Bran Shorts & 30.0 \\
\hline Soyabean Oil & 15.0 \\
\hline Dicalcium Phosphate & 13.5 \\
\hline Limestone & 12.8 \\
\hline Broiler Premix & 7.5 \\
\hline NaCl (salt) & 4.6 \\
\hline Bentonite & 3.0 \\
\hline Luprosil Salt & 0.9 \\
\hline Methionine dl & 0.4 \\
\hline Calculated Chemical Composition(g/kg-1 DM) & \\
\hline Dry Matter* & 889 \\
ME (kcal) & 2.850 \\
\hline Crude Protein* & 224 \\
NDF* & 164 \\
\hline Ca & 10.5 \\
Available P & 4.6 \\
\hline Ca: Available P & 2.3 \\
Lysine & 15.0 \\
Methionine & 4.66 \\
Methionine + Cysteine & 9.16 \\
\hline Tryptophan & 3.55 \\
\hline ME/P & 12.7 \\
\hline Feed Pellet Quality Factor (FPQF) & 3.7 \\
\hline
\end{tabular}

\section{Experimental design}

According to post-hatch feeding time, the ducklings were randomly assigned to four groups viz., T1:3 hours, T2: 24 hours, T3: 36 hours, and T4:48 hours. Each group consisted of 30 birds housed in six cages in a complete randomized design. Treatment one ( T1) received both water and feed three hours post-hatch while the other treatments (T2, T3, and T4) received only water until 24, 36, and 48 hours respectively, had passed. The experiment was conducted for five days which is generally considered the time taken for the total absorption of the yolk. Every day (from Day one to Day five), one duckling from each replicate was randomly selected from each treatment group and the gross and histological studies conducted.

\section{Morphological/Histological studies}

On each day, six ducklings per treatment were randomly selected and individually weighed on an electric scale (OHAUS IR SENSOR). They were then euthanized by cervical dislocation (according to APA 2000 Ethics code), the abdominal cavity was opened and the gastrointestinal tract was excised. The weight of the liver, gizzard, and small intestine were taken using an electronic scale (OHAUS IR SENSOR). The 
length of the three segments of the small intestine (duodenum, jejunum, and ileum) was measured in centimeters using a metric measurement card (marker). The duodenum was measured from the junction of the gizzard to the distal end of the duodenal loop; the jejunum was measured from the distal end of the duodenal loop to the Meckel's diverticulum; while the ileum was measured from the Meckel's diverticulum to the point of attachment of the caeca.

For the histological study, approximately $5 \mathrm{~cm}$ in length of the middle portion of each segment of the small intestine was excised and washed gently in a physiological saline solution to remove the intestinal content. Tissue samples were fixed in $10 \%$ formalin for 48 hours and then processed by routine paraffin embedding (Survana et al., 2013). Tissues were cut transversely with 5-8 $\mu \mathrm{m}$ thickness and stained with Haematoxylin and Eosin (H\&E). The histological slides were examined for histological features using an Olympus BX51 microscope (Olympus, Japan) and photomicrographs and measurements were taken using Olympus cellSens Standard Ver. 1.12 imaging software. The histometrical parameters such as villi length and width, crypt width, and depth and number of goblet cells per villus were measured $(\mu \mathrm{m})$. Villi length was measured from the top of the villi to the beginning of the crypt. Villi width was measured equidistant from the top of the villus to the start of the crypt. The crypt depth was measured from the bottom end of the villi to the base of the crypt. The crypt width is the transverse diameter of the crypt

(Figure 1). Ten measurements were taken per slide and an average taken to give the measurement for each bird. The average number of goblet cells per villi was obtained by counting the number of visible cells on each measured villus (Ganjali et al.2015, 945-955).

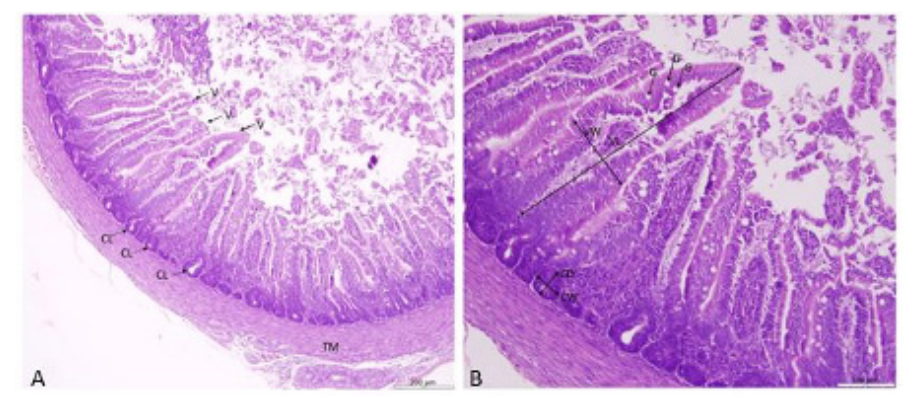

Figure 1 - Showing the cross-section of the duodenum (A\&B) of the 3-day-old duckling fed at 3 hours post-hatch. V-Villi; CL- Crypts of Lieberkühn/Intestinal crypts; TM-Tunica muscularis. VI-Villus length; VW-Villus width; CD-Crypt depth; CW-Crypt width G-Goblet cells.

\section{Statistical Analysis}

The weights of the liver, gizzard, proventriculus, small intestine, the height and width of both the villi and crypt as well as the goblet cells were subjected to a one way Analysis of Variance (ANOVA) test and mean separation (Fisher's pair-wise comparison) was done using the software Minitab release 13 for windows (Minitab software,13). Statements of significance were based on $p<0.05$ which implies the $95 \%$ confidence level.

\section{RESULTS}

Table 2 - Effect of Post -hatch feeding times on the weights of selected structures of the gastrointestinal tract/associated organs of the gastrointestinal tract of Mule ducklings.

\begin{tabular}{|c|c|c|c|c|c|c|}
\hline \multirow[t]{2}{*}{ Parameters (g) } & \multicolumn{4}{|c|}{ Treatment (hours) } & \multirow[t]{2}{*}{ SEM \pm} & \multirow[t]{2}{*}{$p$-Value } \\
\hline & 3 & 24 & 36 & 48 & & \\
\hline \multicolumn{7}{|l|}{ Day 1} \\
\hline Body weight & $48.48^{a}$ & $51.27^{a}$ & $51.55^{a}$ & $47.07^{a}$ & 1.24 & 0.471 \\
\hline Liver & $1.43^{a}$ & $1.28^{a}$ & $1.55^{a}$ & $1.43^{a}$ & 0.31 & 0.620 \\
\hline Gizzard & $1.98^{a}$ & $1.95^{\mathrm{a}}$ & $2.08^{a}$ & $1.93^{a}$ & 0.28 & 0.812 \\
\hline Proventriculus & $0.33^{a}$ & $0.50^{a}$ & $0.30^{a}$ & $0.28^{a}$ & 0.27 & 0.511 \\
\hline Small intestines & $1.05^{a}$ & $1.33^{a}$ & $1.60^{a}$ & $1.35^{a}$ & 0.21 & 0.311 \\
\hline SI g/kg BW & $2.05^{\mathrm{a}}$ & $2.59^{a}$ & $3.12^{\mathrm{a}}$ & $2.87^{\mathrm{a}}$ & 0.28 & 0.223 \\
\hline \multicolumn{7}{|l|}{ Day 2} \\
\hline Body weight & $66.35^{a}$ & $53.30^{b}$ & $56.55^{b}$ & $49.17^{b}$ & 1.20 & 0.001 \\
\hline Liver & $2.28^{a}$ & $1.75^{b}$ & $1.43^{b}$ & $1.38^{b}$ & 0.27 & 0.000 \\
\hline Gizzard & $3.60^{a}$ & $2.73^{a b}$ & $2.08^{a}$ & $2^{a}$ & 0.45 & 0.009 \\
\hline Proventriculus & $0.70^{a}$ & $0.53^{a}$ & $0.43^{a}$ & $0.50^{a}$ & 0.28 & 0.113 \\
\hline Small intestines & $3.15^{a}$ & $1.95^{b}$ & $1.45^{b}$ & $1.36^{b}$ & 0.22 & 0.000 \\
\hline $\mathrm{SI}$ g/kg BW & $4.74^{a}$ & $3.70^{a b}$ & $2.59^{b}$ & $2.76^{b}$ & 0.30 & 0.008 \\
\hline \multicolumn{7}{|l|}{ Day 3} \\
\hline Body weight & $81.13^{a}$ & $80.03^{a}$ & $73.08^{a}$ & $59^{b}$ & 1.21 & 0.000 \\
\hline Liver & $3.18^{a}$ & $3.00^{a}$ & $2.52^{\mathrm{ab}}$ & $1.75^{b}$ & 0.41 & 0.006 \\
\hline Gizzard & $5.08^{a}$ & $4.10^{a b}$ & $3.50^{b}$ & $3.45^{b}$ & 0.43 & 0.004 \\
\hline Proventriculus & $0.75^{a}$ & $0.93^{a}$ & $0.90^{\mathrm{a}}$ & $0.73^{a}$ & 0.26 & 0.396 \\
\hline Small intestines & $3.90^{a}$ & $3.77^{\mathrm{a}}$ & $3.33^{a b}$ & $2.93^{b}$ & 0.19 & 0.005 \\
\hline SI g/kg BW & $4.80^{a}$ & $4.73^{a}$ & $4.56^{a}$ & $4.97^{a}$ & 0.22 & 0.643 \\
\hline
\end{tabular}

Parameters with a similar superscript in different rows indicates differences between means are not significant $(p<0.05)$. 
Table 2 continued - Effect of Post- hatch feeding times on the weights of selected structures of the gastrointestinal tract/ associated organs of the gastrointestinal tract of Mule ducklings.

\begin{tabular}{|c|c|c|c|c|c|c|}
\hline \multirow[t]{2}{*}{ Parameters (g) } & \multicolumn{4}{|c|}{ Treatment (hours) } & \multirow[t]{2}{*}{ SEM \pm} & \multirow[t]{2}{*}{$p$-Value } \\
\hline & 3 & 24 & 36 & 48 & & \\
\hline \multicolumn{7}{|l|}{ Day 4} \\
\hline Body weight & $85.63^{a}$ & $77.75^{\mathrm{a}}$ & $80.05^{a}$ & $61.42^{b}$ & 1.16 & 0.000 \\
\hline Liver & $4.23^{a}$ & $4.05^{a}$ & $4.35^{\mathrm{a}}$ & $3.40^{a}$ & 0.45 & 0.174 \\
\hline Gizzard & $6.15^{\mathrm{a}}$ & $5.00^{a b}$ & $5.15^{b}$ & $3.78^{c}$ & 0.42 & 0.000 \\
\hline Proventriculus & $0.68^{a}$ & $0.85^{a}$ & $0.80^{a}$ & $0.75^{a}$ & 0.27 & 0.728 \\
\hline Small intestines & $5.40^{a}$ & $4.40^{a b}$ & $4.95^{b}$ & $3.38^{c}$ & 0.20 & 0.000 \\
\hline SI g/kg BW & $6.33^{\mathrm{a}}$ & $5.66^{\mathrm{a}}$ & $6.18^{\mathrm{a}}$ & $5.50^{\mathrm{a}}$ & 0.24 & 0.108 \\
\hline \multicolumn{7}{|l|}{ Day 5} \\
\hline Body weight & $99.65^{\mathrm{a}}$ & $81.28^{a b}$ & $88.70^{b}$ & $74.75^{b}$ & 1.51 & 0.002 \\
\hline Liver & $6.05^{\mathrm{a}}$ & $3.55^{\mathrm{a}}$ & $4.35^{a}$ & $3.68^{a}$ & 0.78 & 0.242 \\
\hline Gizzard & $7.33^{a}$ & $5.53^{a b}$ & $6.10^{b}$ & $4.95^{b}$ & 0.51 & 0.006 \\
\hline Proventriculus & $1.43^{\mathrm{a}}$ & $0.90^{\mathrm{a}}$ & $1.33^{\mathrm{a}}$ & $1.38^{\mathrm{a}}$ & 0.31 & 0.095 \\
\hline Small intestines & $6.42^{\mathrm{a}}$ & $4.73^{\mathrm{a}}$ & $6.33^{a}$ & $4.93^{\mathrm{a}}$ & 0.54 & 0.345 \\
\hline $\mathrm{SI}$ g/kg BW & $6.54^{\mathrm{a}}$ & $5.81^{\mathrm{a}}$ & $7.17^{\mathrm{a}}$ & $6.56^{\mathrm{a}}$ & 0.44 & 0.747 \\
\hline
\end{tabular}

SI- Small Intestine. Parameters with a similar superscript in different rows indicates differences between means are not significant $(p<0.05)$.

Table 3 - Effect of post-hatch feeding times on the length $(\mathrm{cm})$ of the intestinal segments of Mule ducklings.

\begin{tabular}{|c|c|c|c|c|c|c|}
\hline Length of S.I $(\mathrm{cm})$ & & Trea & urs) & & $\operatorname{SEM}( \pm)$ & $p$-value \\
\hline Day 1 & 3 & 24 & 36 & 48 & & \\
\hline Duodenum & $11.25^{a}$ & $8.93^{\mathrm{ab}}$ & $8.35^{b}$ & $7.83^{b}$ & 0.65 & 0.011 \\
\hline Jejunum & $19.25^{a}$ & $24^{\mathrm{ab}}$ & $37.75^{\mathrm{ab}}$ & $32.13^{b}$ & 1.64 & 0.030 \\
\hline Ileum & $12.00^{b c}$ & $8.38^{c}$ & $14.08^{\mathrm{ab}}$ & $18.13^{\mathrm{a}}$ & 0.90 & 0.001 \\
\hline Day 2 & & & & & & \\
\hline Duodenum & $10.50^{a}$ & $9.10^{a}$ & $8.93^{a}$ & $9.33^{a}$ & 0.76 & 0.586 \\
\hline Jejunum & $23.88^{a}$ & $27.13^{a}$ & $23.13^{a}$ & $21.93^{a}$ & 1.08 & 0.234 \\
\hline Ileum & $13.75^{\mathrm{a}}$ & $10.88^{a}$ & $18.10^{a b}$ & $19.25^{b}$ & 0.96 & 0.004 \\
\hline Day 3 & & & & & & \\
\hline Duodenum & $10.48^{a}$ & $10.28^{a}$ & $12.13^{a}$ & $11.60^{a}$ & 1.15 & 0.837 \\
\hline Jejunum & $25.50^{\mathrm{a}}$ & $24.35^{a}$ & $18.13^{a}$ & $22.70^{\mathrm{a}}$ & 1.11 & 0.093 \\
\hline Ileum & $24.25^{\mathrm{a}}$ & $22.82^{\mathrm{a}}$ & $19.43^{\mathrm{a}}$ & $17.75^{\mathrm{a}}$ & 1.06 & 0.102 \\
\hline Day 4 & & & & & & \\
\hline Duodenum & $11.40^{\mathrm{a}}$ & $8.18^{a}$ & $10.28^{a}$ & $10.18^{a}$ & 0.94 & 0.425 \\
\hline Jejunum & $33.10^{\mathrm{a}}$ & $27.05^{\mathrm{ab}}$ & $28.23^{a b}$ & $24.73^{b}$ & 0.99 & 0.011 \\
\hline Ileum & $31.02^{\mathrm{a}}$ & $26.40^{\mathrm{ab}}$ & $25.38^{b}$ & $23.70^{\mathrm{b}}$ & 0.95 & 0.013 \\
\hline Day 5 & & & & & & \\
\hline Duodenum & $13.38^{a}$ & $12.05^{a}$ & $11.80^{a}$ & $11.60^{a}$ & 0.72 & 0.399 \\
\hline Jejunum & $32.80^{\mathrm{a}}$ & $30.33^{a}$ & $28.43^{a}$ & $27.70^{a}$ & 1.08 & 0.219 \\
\hline Ileum & $32.98^{a}$ & $30.30^{a}$ & $28.45^{\mathrm{a}}$ & $25.85^{\mathrm{a}}$ & 1.13 & 0.116 \\
\hline
\end{tabular}

Parameters with similar superscript in different rows indicates differences between means are not significant $(p<0.05)$.

Table 4 - Effects of Post-hatch feeding on the Morphological Parameters of Duodenum in Mule ducklings.

\begin{tabular}{|c|c|c|c|c|c|c|}
\hline \multirow{2}{*}{$\begin{array}{l}\text { Parameter }(\mu \mathrm{m}) \\
\text { Day } 1\end{array}$} & \multicolumn{4}{|c|}{ Treatment } & \multirow[t]{2}{*}{ SEM \pm} & \multirow[t]{2}{*}{$p$-value } \\
\hline & 3 hours & 24 hours & 36 hours & 48 hours & & \\
\hline \multicolumn{7}{|l|}{ Duodenum } \\
\hline Villus height & $21.01^{a}$ & $36.85^{a}$ & $33.07^{a}$ & $32.42^{b}$ & 0.94 & 0.001 \\
\hline Villus width & $5.29^{\mathrm{a}}$ & $5.84^{\mathrm{ab}}$ & $7.71^{\mathrm{b}}$ & $4.80^{\mathrm{b}}$ & 0.46 & 0.008 \\
\hline Crypt height & $5.06^{a}$ & $3.46^{\mathrm{ab}}$ & $5.74^{\mathrm{ab}}$ & $4.03^{b}$ & 0.44 & 0.027 \\
\hline Crypt width & $1.79^{\mathrm{a}}$ & $1.94^{b}$ & $4.44^{b}$ & $2.52^{b}$ & 0.33 & 0.000 \\
\hline Goblet cells & $4.00^{\mathrm{a}}$ & $5.90^{\mathrm{a}}$ & $2.50^{\mathrm{a}}$ & $3.70^{\mathrm{a}}$ & 0.64 & 0.239 \\
\hline \multicolumn{7}{|l|}{ Day 2} \\
\hline Villus height & $27.47^{a}$ & $31.03^{b}$ & $77.31^{c}$ & $59.01^{c}$ & 1.31 & 0.578 \\
\hline Villus width & $13.80^{\mathrm{a}}$ & $10.9^{b}$ & $4.58^{c}$ & $5.49^{c}$ & 0.50 & 0.000 \\
\hline Crypt height & $14.45^{\mathrm{a}}$ & $14.07^{\mathrm{a}}$ & $17.45^{\mathrm{a}}$ & $15.35^{\mathrm{a}}$ & 0.61 & 0.140 \\
\hline Crypt width & $20.21^{\mathrm{a}}$ & $17.78^{a}$ & $12.76^{b}$ & $8.79^{c}$ & 0.61 & 0.000 \\
\hline Goblet cells & $5.00^{\mathrm{a}}$ & $6.56^{a}$ & $6.10^{\mathrm{a}}$ & $2.91^{\mathrm{a}}$ & 0.71 & 0.278 \\
\hline \multicolumn{7}{|l|}{ Day 3} \\
\hline Villus height & $74.10^{\mathrm{a}}$ & $52.66^{b}$ & $50.24^{b}$ & $51.65^{b}$ & 1.10 & 0.000 \\
\hline Villus width & $19.13^{\mathrm{a}}$ & $31.34^{\mathrm{ab}}$ & $13.95^{b}$ & $14.98^{b}$ & 1.20 & 0.024 \\
\hline Crypt height & $18.80^{\mathrm{a}}$ & $23.21^{b}$ & $17.40^{b}$ & $18.16^{\mathrm{b}}$ & 1.05 & 0.003 \\
\hline Crypt width & $14.44^{\mathrm{a}}$ & $25.88^{b}$ & $10.20^{b}$ & $12.09^{\mathrm{b}}$ & 0.92 & 0.000 \\
\hline Goblet cells & $10.33^{a}$ & $8.00^{a}$ & $7.90^{\mathrm{a}}$ & $7.36^{a}$ & 0.25 & 0.000 \\
\hline
\end{tabular}

Parameters with a similar superscript in different rows indicates differences between means are not significant $(p<0.05)$. 
Table 4 continued - Effects of Post-hatch feeding on the Morphological Parameters of Duodenum in Mule ducklings.

\begin{tabular}{|c|c|c|c|c|c|c|}
\hline Parameter $(\mu \mathrm{m})$ & \multicolumn{4}{|c|}{ Treatment } & SEM \pm & $p$-value \\
\hline \multicolumn{7}{|l|}{ Day 4} \\
\hline Duodenum & 3 hours & 24 hours & 36 hours & 48 hours & & \\
\hline Villus height & $97.7^{\mathrm{a}}$ & $106.71^{\mathrm{a}}$ & $145.36^{\mathrm{a}}$ & $109.38^{a}$ & 2.37 & 0.178 \\
\hline Villus width & $163.7^{\mathrm{a}}$ & $113.15^{b}$ & $24.84^{c}$ & $22.16^{c}$ & 2.10 & 0.000 \\
\hline Crypt height & $4.07^{a}$ & $117.74^{b}$ & $17.29^{b}$ & $14.11^{b}$ & 1.68 & 0.000 \\
\hline Crypt width & $36.6^{a}$ & $116.45^{b}$ & $11.80^{b}$ & $14.11^{b}$ & 1.56 & 0.000 \\
\hline Goblet cells & $5.20^{a}$ & $0.30^{a}$ & $2.80^{\mathrm{ab}}$ & $6.70^{b}$ & 0.66 & 0.005 \\
\hline \multicolumn{7}{|l|}{ Day 5} \\
\hline Villus height & $120.71^{\mathrm{a}}$ & $102.32^{a b}$ & $85.68^{b}$ & $38.69^{c}$ & 1.40 & 0.000 \\
\hline Villus width & $126.46^{a}$ & $122.17^{a}$ & $117.11^{\mathrm{a}}$ & $123.99^{b}$ & 0.84 & 0.000 \\
\hline Crypt height & $17.57^{a}$ & $16.11^{\mathrm{a}}$ & $5.83^{a}$ & $15.82^{b}$ & 0.67 & 0.000 \\
\hline Crypt width & $13.31^{\mathrm{a}}$ & $10.00^{b}$ & $3.29^{b}$ & $9.60^{c}$ & 0.46 & 0.000 \\
\hline Goblet cells & $9.60^{a}$ & $3.30^{b}$ & $4.30^{b}$ & $6.00^{c}$ & 0.90 & 0.252 \\
\hline
\end{tabular}

Parameters with a similar superscript in different rows indicates differences between means are not significant $(p<0.05)$.

Table 5 - Effects of Post-hatch feeding on the Morphological Parameters of Jejunum in Mule ducklings.

\begin{tabular}{|c|c|c|c|c|c|c|}
\hline \multirow{2}{*}{$\begin{array}{l}\text { Parameter }(\mu \mathrm{m}) \\
\text { Day } 1\end{array}$} & \multicolumn{4}{|c|}{ Treatment } & \multirow[t]{2}{*}{ SEM \pm} & \multirow[t]{2}{*}{$p$-value } \\
\hline & 3 hours & 24 hours & 36 hours & 48 hours & & \\
\hline \multicolumn{7}{|l|}{ Jejunum } \\
\hline Villus height & $19.29^{a}$ & $24.28^{a b}$ & $26.85^{a b}$ & $23.47^{b}$ & 0.77 & 0.029 \\
\hline Villus width & $7.38^{a}$ & $4.30^{b}$ & $5.19^{b}$ & $5.66^{b}$ & 0.37 & 0.000 \\
\hline Crypt height & $3.66^{a}$ & $3.39^{a}$ & $4.12^{a}$ & $2.74^{\mathrm{a}}$ & 0.36 & 0.073 \\
\hline Crypt width & $2.66^{a}$ & $1.94^{\mathrm{ab}}$ & $1.88^{\mathrm{b}}$ & $1.85^{b}$ & 0.26 & 0.021 \\
\hline Goblet cells & $1.80^{\mathrm{a}}$ & $5.30^{\mathrm{ab}}$ & $4.10^{\mathrm{ab}}$ & $2.60^{b}$ & 0.57 & 0.048 \\
\hline \multicolumn{7}{|l|}{ Day 2} \\
\hline Villus height & $73.85^{a}$ & $32.66^{b}$ & $30.82^{b}$ & $36.58^{b}$ & 1.14 & 0.000 \\
\hline Villus width & $28.24^{a}$ & $28.23^{a}$ & $21.79^{b}$ & $21.18^{b}$ & 0.85 & 0.021 \\
\hline Crypt height & $12.12^{\mathrm{a}}$ & $5.52^{b}$ & $9.57^{b}$ & $8.98^{c}$ & 0.48 & 0.000 \\
\hline Crypt width & $8.35^{\mathrm{a}}$ & $3.89^{b}$ & $8.84^{b}$ & $4.87^{b}$ & 0.47 & 0.000 \\
\hline Goblet cells & $3.60^{a}$ & $7.50^{b}$ & $2.50^{b}$ & $0.80^{b}$ & 0.54 & 0.000 \\
\hline \multicolumn{7}{|l|}{ Day 3} \\
\hline Villus height & $95.03^{a}$ & $102.32^{\mathrm{ab}}$ & $79.50^{a b}$ & $103.23^{b}$ & 1.45 & 0.030 \\
\hline Villus width & $23.38^{a}$ & $22.17^{\mathrm{a}}$ & $22.23^{a}$ & $25.07^{a}$ & 0.76 & 0.574 \\
\hline Crypt height & $16.46^{a}$ & $16.11^{\mathrm{a}}$ & $14.82^{a}$ & $14.25^{\mathrm{a}}$ & 0.64 & 0.494 \\
\hline Crypt width & $8.32^{a}$ & $9.98^{a b}$ & $7.88^{a b}$ & $8.93^{b}$ & 0.44 & 0.056 \\
\hline Goblet cells & $1.10^{a}$ & $3.10^{a}$ & $3.70^{a}$ & $4.80^{a}$ & 0.62 & 0.131 \\
\hline \multicolumn{7}{|l|}{ Day 4} \\
\hline Villus height & $126.10^{a}$ & $144.53^{\mathrm{ab}}$ & $101.62^{b}$ & $69.94^{c}$ & 0.46 & 0.000 \\
\hline Villus width & $31.71^{a}$ & $25.42^{\mathrm{a}}$ & $28.74^{a b}$ & $20.10^{b}$ & 0.80 & 0.001 \\
\hline Crypt height & $17.34^{a}$ & $20.07^{a b}$ & $15.95^{a b}$ & $13.89^{b}$ & 0.66 & 0.009 \\
\hline Crypt width & $12.83^{a}$ & $14.74^{\mathrm{ab}}$ & $10.53^{b c}$ & $9.08^{c}$ & 0.55 & 0.000 \\
\hline Goblet cells & $2.50^{a}$ & $0.30^{a}$ & $1.50^{a}$ & $2.20^{a}$ & 1.03 & 0.065 \\
\hline \multicolumn{7}{|l|}{ Day 5} \\
\hline Villus height & $150.51^{\mathrm{a}}$ & $106.71^{\mathrm{a}}$ & $157.8^{b}$ & $79.38^{b}$ & 1.62 & 0.000 \\
\hline Villus width & $160.1^{b}$ & $113.15^{b}$ & $158.6^{b}$ & $19.91^{b}$ & 1.62 & 0.000 \\
\hline Crypt height & $181.15^{\mathrm{a}}$ & $117.74^{\mathrm{a}}$ & $156.7^{b}$ & $14.53^{c}$ & 1.72 & 0.000 \\
\hline Crypt width & $176.05^{\mathrm{a}}$ & $116.45^{a}$ & $156.7^{b}$ & $7.50^{c}$ & 1.69 & 0.000 \\
\hline Goblet cells & $17.80^{a}$ & $17.80^{a}$ & $16.70^{a}$ & $7.00^{b}$ & 1.52 & 0.000 \\
\hline
\end{tabular}

Parameters ${ }^{\text {ab }}$ with similar superscript in different rows indicate differences between means are not significant $(p<0.05)$. 
Table 6 - Effects of Post-hatch feeding on the Morphological Parameters of lleum in Mule ducklings.

\begin{tabular}{|c|c|c|c|c|c|c|}
\hline \multirow{2}{*}{$\begin{array}{l}\text { Parameter }(\mu \mathrm{m}) \\
\text { Day } 1\end{array}$} & \multicolumn{4}{|c|}{ Treatment } & \multirow[t]{2}{*}{ SEM \pm} & \multirow[t]{2}{*}{$p$-value } \\
\hline & 3 hours & 24 hours & 36 hours & 48 hours & & \\
\hline \multicolumn{7}{|l|}{ Ileum } \\
\hline Villus height & $23.92^{\mathrm{a}}$ & $30.13^{a b}$ & $31.73^{b c}$ & $24.36^{c}$ & 0.74 & 0.001 \\
\hline Villus width & $6.66^{a}$ & $4.06^{a b}$ & $5.49^{a b}$ & $4.89^{b}$ & 0.46 & 0.034 \\
\hline Crypt height & $3.95^{\mathrm{a}}$ & $2.72^{a}$ & $4.30^{a}$ & $3.16^{\mathrm{a}}$ & 0.39 & 0.057 \\
\hline Crypt width & $1.95^{\mathrm{a}}$ & $1.66^{\mathrm{ab}}$ & $2.41^{b}$ & $1.66^{b}$ & 0.22 & 0.002 \\
\hline Goblet cells & $1.30^{\mathrm{a}}$ & $3.20^{\mathrm{a}}$ & $3.20^{\mathrm{a}}$ & $2.10^{\mathrm{a}}$ & 0.48 & 0.143 \\
\hline \multicolumn{7}{|l|}{ Day 2} \\
\hline Villus height & $45.78^{a}$ & $20.59^{a b}$ & $32.36^{a b}$ & $34.60^{b}$ & 1.26 & 0.004 \\
\hline Villus width & $6.76^{a}$ & $5.93^{b}$ & $4.78^{b}$ & $6.43^{b}$ & 1.46 & 0.000 \\
\hline Crypt height & $20.24^{a}$ & $4.31^{b}$ & $3.91^{b}$ & $3.48^{b}$ & 0.99 & 0.000 \\
\hline Crypt width & $20.99^{a}$ & $3.05^{b}$ & $2.17^{b}$ & $2.14^{b}$ & 0.84 & 0.000 \\
\hline Goblet cells & $5.80^{\mathrm{a}}$ & $2.40^{\mathrm{a}}$ & $3.80^{\mathrm{a}}$ & $6.10^{a}$ & 0.622 & 0.075 \\
\hline \multicolumn{7}{|l|}{ Day 3} \\
\hline Villus height & $78.56^{a}$ & $76.31^{\text {a }}$ & $59.85^{a}$ & $80.65^{\text {a }}$ & 1.42 & 0.057 \\
\hline Villus width & $24.25^{a}$ & $23.73^{a}$ & $20.97^{a}$ & $19.59^{a}$ & 0.71 & 0.086 \\
\hline Crypt height & $13.59^{a}$ & $15.13^{a b}$ & $10.94^{b}$ & $11.11^{b}$ & 0.60 & 0.015 \\
\hline Crypt width & $90.66^{a}$ & $9.69^{a}$ & $6.46^{\mathrm{ab}}$ & $7.75^{b}$ & 0.50 & 0.006 \\
\hline Goblet cells & $4.30^{a}$ & $4.80^{a}$ & $0.70^{a b}$ & $3.20^{\mathrm{b}}$ & 0.57 & 0.019 \\
\hline Day 4 & 3 hours & 24 hours & 36 hours & 48 hours & & \\
\hline Villus height & $91.20^{a}$ & $71.73^{b}$ & $53.01^{c}$ & $59.45^{c}$ & 1.05 & 0.000 \\
\hline Villus width & $27.10^{a}$ & $27.29^{a}$ & $25.11^{\mathrm{a}}$ & $20.46^{a}$ & 0.80 & 0.043 \\
\hline Crypt height & $20.25^{a}$ & $20.07^{a}$ & $11.11^{b}$ & $14.81^{b}$ & 0.64 & 0.000 \\
\hline Crypt width & $15.94^{a}$ & $12.22^{b}$ & $7.21 \mathrm{bc}$ & $9.16^{c}$ & 0.54 & 0.000 \\
\hline Goblet cells & $5.20^{\mathrm{a}}$ & $11.40^{\mathrm{ab}}$ & $5.90^{\mathrm{ab}}$ & $1.60^{b}$ & 0.78 & 0.004 \\
\hline \multicolumn{7}{|l|}{ Day 5} \\
\hline Villus height & $181.7^{a}$ & $93.4^{\mathrm{a}}$ & $150.5^{a}$ & $142.29^{b}$ & 0.59 & 0.000 \\
\hline Villus width & $182.1^{\mathrm{a}}$ & $95.9^{a}$ & $149.3^{a}$ & $141.9^{b}$ & 1.98 & 0.000 \\
\hline Crypt height & $103.9^{a}$ & $93.7^{a b}$ & $150.1^{a b}$ & $141.3^{b}$ & 2.12 & 0.008 \\
\hline Crypt width & $104.8^{a}$ & $92.6^{a b}$ & $148.1^{\mathrm{ab}}$ & $140.2^{b}$ & 2.11 & 0.009 \\
\hline Goblet cells & $1.90^{\mathrm{a}}$ & $0.30^{a}$ & $0.000^{a}$ & $1.30^{\circ}$ & 0.43 & 0.049 \\
\hline
\end{tabular}

Parameters with a similar superscript in different rows indicates differences between means are not significant $(p<0.05)$.

\section{Accessory Organs}

The results revealed that on Day 2 and Day 3, the body weight $(p<0.05)$ and liver $(p<0.05)$ and gizzard $(p<0.05)$ weight differed significantly between the four-post-hatch feeding times. However, on Days 4 and 5 , the body weight $(p=0.002)$ and gizzard weight $(p<0.05)$ alone were significantly different. It was also observed that on Days two, three, and four, the small intestine weight $(p<0.05)$ and the relative weight of the small intestine $(p<0.05)$ differed significantly between treatment groups (Table.2)

\section{Length of the small intestine}

The length of all three segments of the small intestine was significantly affected by the feeding times $(p<0.05)$ in Day one. However, on Day two, only the length of the ileum showed significant differences among the post-hatch feeding groups $(p<0.05)$. Post hatch feeding times on Days three and five did not significantly affect the small intestines segment of mule ducks.

\section{Histometrical parameters}

A constant increase in the villi height was observed throughout the experimental period. All parameters of the duodenum except the goblet cells were affected by post-hatch feeding times at Day one. Jejunal villi height, villi width, crypt height, and goblet cells were also affected by post-hatch feeding times. For the ileum the villi height, width, and crypt width showed significant differences between treatments on Day one. Similarly, on Days two all jejunal parameters $(p>0.05)$ were influenced by treatment except for villi height. All histometrical parameters of the duodenum except the goblets cells of the ileum differed significantly between treatment groups on Day three $(p<0.05)$; respectively. While for the jejunum only the villi height was significantly affected $(p<0.05)$ and the crypt width and goblet cells were affected by post-hatch feeding times 
$(p<0.05)$. At the end of the experimental period (Day five), all histometrical parameters were significantly affected by treatment times with ducklings belonging to the group fed 3 hours post-hatch, showing the highest for all sections of the jejunal observed.

\section{DISCUSSION}

The presence of exogenous feed in the gastrointestinal tract plays a fundamental role in initiating growth during the early post-hatch (Maiorka et al. 2003). In this present study, the bodyweight of ducklings fed at the 3 hours post-hatch was significantly higher $(p<0.05)$ than those fed at 24, 36, and 48 hours post-hatch which concurs with the reports of Ganjali et al. (2015) in broiler chicks and Loudon et al. (2011) in Pekin ducklings.

\section{Post hatch feeding Effect on Small Intestine Growth}

Early feed consumption provides the nutrients that are complementary to the yolk nutrients and triggers rapid growth rate in birds Geyra et al. (2001a).

The observed lower body weight in the groups fed 24,36 , and 48 h during the first five days of life in the present study is consistent with reports of Saki 2005 in which delayed access to feed decreased broiler posthatch performance. Tabeidan (2011) attributed the low body weight with increasing delayed fed period (24, 36, and 48h) to lower feed intake and poor development of the GIT of ducklings. The experiment revealed that the bodyweight increased daily regardless of the post-hatch feeding times.

\section{Accessory organs and relative weight}

Post hatch the gastrointestinal tract plays a fundamental role in initiating growth (Maiorka et al., 2003). The growth of the GIT occurs as a result of an increase in the surface area for digestion and absorption. It also includes a proliferation in the length of the intestines, villus height, density, and the number of enterocytes and goblet cells (Imondi \& Bird, 1966; Baranyiova, 1972; Baranyiova \& Holman, 1976). In this present study ducklings fed at 3 hours post-hatch were seen to have heavier GIT organ weights than the others fed at 24, 36, and 48 hours on all five days. This observation may be attributed to the availability of energy and protein at an early stage which was used for the development of the intestine. The lower GIT organ weights observed in the other treatment groups (24, 36, and 48 hours) may be as a result of feed deprivation (Maiorka et al., 2003; Ganjali et al., 2015).
The weights of the gizzard and small intestines of ducklings from this present study differed between treatment groups on Days two and four; while on Day five only the gizzard weight exhibited significant difference $(p<0.05)$ between treatments. The daily increase of liver weight was higher in ducklings fed at 3 and 24 hours than those fed at 36 and 48 hours. It was also observed that on Days two and three, the liver, gizzard, and small intestines weights were significantly influenced by the treatment. These differences can be attributed to the inability of the residual yolk sac to supply sufficient nutrients requires for the growth of the small intestine and proventriculus as demonstrated in broiler chicks and poults (Murakami et al., 1992; Noy \& Sklan, 1997). These results confirmed the fact that the overall growth of the organs of the GIT is stimulated by the presence of exogenous feed in the lumen of the SI (Bigot et al., 2003; Moran, 1990). Therefore, earlier feeding times can be beneficial to Mule ducks because it stimulates growth and increases growth rate of the GIT.

\section{Length of the small intestine}

The small intestine serves an important function in nutrient digestion and absorption and overall health of the bird and is composed of three parts: duodenum, jejunum, and ileum, which aids in the further enzymatic breakdown, digestion, and absorption of nutrients (Sklan, 2001). The rapid development post-hatch of the gastrointestinal tract in hatchlings is a result of an increase in both the length and diameter of the intestine (Przyslalski, 1987a). On Day 5 ducklings fed at 3 hours post-hatch had the highest relative duodenal length $(10.5,2.1$ and $1.7 \%$; respectively) even though there was no significant difference observed $(p>0.05)$ between treatment groups as observed in broiler chicks (Maiorka et al., 2003; Imondi \& Bird, 1966; Baranyiova, 1972; Baranyiova \& Holman, 1976). On Days one and four the time of post-hatch feeding was noted to significantly affect the length of the jejunum $(p<0.05)$. Additionally, on Day five ducklings fed 3 hours posthatch had the highest jejunal length. The length of the jejunum fluctuated over the five days for all treatment groups. These fluctuations were supported by the reports of several authors who have highlighted that post-hatch the gastrointestinal tract does not develop at a constant rate (Uni et al., 1999; lji et al., 2001a).

The lleal length differed between treatment groups $(p<0.05)$ on Days one, two, and four (Table 6). The length of the ileum was observed to be the highest in the group of ducklings fed at 48 hours compared to those fed at 3, 24, and 36 hours on Days one and two. 
These results contrasted with the reports of Loudon et al. (2011) on white Pekin ducklings where higher lleal lengths were recorded in birds fed immediately post-hatch. However, the ileal length was the highest on Day four, for the group fed 3 hours post-hatch as reported by Loudon et al. (2011). The fluctuations in the present study could be possibly due to the effect of the presence of water which was given ad libitum.

The total absolute lengths of the small intestine in the current study ranged between 41.31-60.18, 47.77-50.51, and 58.61-75.52 cm for Days one, two, and four; respectively. These results when compared to previous studies showed that the SI length was similar to broiler chickens (Ganjali et al., 2015). The relative jejunal length was highest in the present study, followed by ileal length; this trend observed was similar to the findings of Applegate \& Lilburn (1999) for turkey poults. These authors highlighted that the increase in the relative length of the jejunum and ileum occurs at a faster rate than the duodenum up to five days. However, it was reported by several authors that the developmental pattern varies significantly between intestinal segments particularly the duodenum and jejunum in chicks (Raheja et al., 1977; Sulistiyanto et al., 1999; Batal \& Parsons, 2002; Sklan, 2003).

\section{Histometrical parameters}

The rapid development of the GIT in hatchlings has been attributed to a significant increase in the length and diameter of the intestine together with an increase in the folds of the villi (Przyslalski, 1987a). There is a correlation between the timing of the introduction of feed and the feed intake of chicks on the growth of the gastrointestinal tract (Noy \& Sklan, 1997). The results obtained from this current study showed that the villi height and width, crypt height and depth but not goblet cells on Day one of the duodenum were affected by post-hatch feeding times $(p<0.05)$.

Sklan (2001) reported that the duodenal villus height increased two-fold during the first two days of post-hatch and reached a plateau at approximately six to eight days in broiler chicks. Ducklings belonging to the group fed at 3 hours post-hatch showed a similar increase to broiler chicks in the duodenal villi height (Sklan, 2001). However, the duodenal villi height of ducklings belonging to the groups fed at 24, 36 and 48 hours post-hatch in this present study did not increase two-fold during the first two days post-hatch. On Day two, the duodenal villi height, width, and crypt width were affected by post-hatch feeding times $(p<0.05)$. This may be due to excessive cell death and a reduction in cell renewal; thus giving rise to an unbalanced cell turnover (Yamaichi et al., 1996).

The villus height increased constantly over the five-day experimental period. However, a fluctuation was noted to have occurred in the growth of the villi width, crypt height, and width and goblet cells between Days one and five. This pattern was mirrored morphometrically by the fluctuation in the growth of the Duodenum over the five days. This histological modification may be related to the availability and nature of the nutrients within the intestine, thus resulting in the small villi undergoing compensatory enlargement due to the intestine having elevated nutrient levels for two to five days (Imondi \& Bird, 1965; Isshiki, 1989). Despite the fluctuation in growth at the end of the experimental period, all parameters showed an increase in growth. This increase in growth reflects a change in the reliance of yolk sac nutrients to solid feed within the first five days post-hatch (Govaerts et al., 2000).

The absorptive capacity of the small intestines of birds is dependent on the growth of the villi (Wijtten et al., 2012). On day five, the ducklings belonging to the 3 hours post-hatch fed group showed increases for all parameters compared to those fed at 24, 36, and 48 hours post-hatch. According to Gonzales et al. (2003) delayed access to feed has been reported to hurt the growth and development of the gastrointestinal tract in birds. The retardation of growth in the villi and crypt of the duodenum of ducklings fed at 24,36 , and 48 hours in this current study is as a result of a decrease in the process of cell proliferation, migration, and extrusion which are responsible for growth (Gonzales et al., 2003).

Delayed access to feed during the first two days post-hatch can lead to an interruption of growth processes. This may include a reduction in duodenal and jejunal crypt size, crypt proliferation, and enterocyte migration rate (Geyra et al., 2001a). From the current study, it was observed that the Jejunal villi height increased constantly throughout the five days. It was also noted that ducklings fed at 3 hours posthatch showed a four-fold increase in the villi height on Day two only compared to those fed at 24,36 , and 48 hours. This increase may be explained by the early presence of exogenous feed in the gut which stimulated the development of the small intestines of early post-hatch fed ducklings (Imondi \& Bird, 1966; Baranyiova, 1972; Baranyiova \& Holman, 1976).

On Day two villi and crypt height, crypt width, and goblet cells were significantly influenced $(p<0.05)$ by treatments $(3,24,36$, and 48 hours). However, on Day 
three no significant effect ( $p>0.005)$ was observed on all jejunal parameters for all treatments; this result was not in agreement with Zarghi (2005) for quails.

At the end of the experimental period, the jejunal parameters of the group fed at 3 hours post-hatch were higher than those fed at 24, 36, and 48 hours posthatch. According to (Noy \& Sklan, 1998b), feeding early post-hatch stimulates the morphological development of the small intestine of broiler chickens. The high villi height, villi width, and crypt width in ducklings fed 3 hours post-hatch may be attributable to the access to food immediately after hatch which has been observed to result in the more rapid development of the intestine during the immediate post-hatch period (Yegani \& Korver, 2008).

The ducklings fed at 3 hours post-hatch from the current study showed a two-fold increase in the ileal villi height in the first 24 hours (on day two) compared to the ducklings fed at 24, 36, and 48 hours. The major increase in the villi height of ducklings may be associated with the early presence of feed in the GIT which stimulated the growth of the villi height (Loudon et al., 2011). At the end of the experimental period (On day five) ducklings fed at 3 hours post-hatch had the highest villi height and width, crypt height and width as well as goblet cells when compared to ducklings fed at 24, 36, and 48 hours post-hatch. Contrastingly, Ganjali et al. (2015) revealed that the opposite occurred at the end of their experimental period; however, their experimental period ended at 12 days post-hatch in comparison to five days used in the present study.

Previous studies showed that larger villus exists in the duodenum than in the jejunum and ileum of broiler chicks (Uni et al., 1998). However, results from this current experiment did not coincide with those obtained by Uni et al. (1998); this variation noted may be attributable to the difference between species.

Individual crypts complete development approximately 48 hours post-hatch and crypt number per villi reaches a peak and remains stable at approximately 72 hours post-hatch (lji et al., 2001a). However, this scenario was not observed in the crypts of ducklings for this current study as it was increased throughout the experiment period. lji et al. (2001a) stated that the depth of crypts varies among GIT segments, similarly, the ducklings for this study showed a variation in the width of crypts between the intestinal segments during the five-day experimental period. The results strongly supported the literature that an increase in the crypt height influenced the growth of the GIT; thus making the ducks efficient in utilizing feed as reflected in the body weight.

\section{CONCLUSION AND RECOMMENDATIONS}

Of all four treatments, feeding at 3 hours posthatch showed superior body weight, heavier GIT organ weights, and highest duodenal length, than other feeding times. The villi height of the three segments of the GIT increases continuously regardless of the post-hatch fed times. Though fluctuations on various parameters were noted in the present study, it was concluded that the mule ducklings followed the pattern of growth and development similar to the broiler chicks. Based on our study results, it is recommended that the earliest possible post-hatch feeding have to be done which in turn will promote the growth and development of the GIT faster than the delayed post-hatch feeding.

\section{ACKNOWLEDGMENTS}

Authors thank Mr. Gerald Chandoo for his assistance with histological work, the workers at the UWI Field Station for physical support.

\section{REFERENCE}

Applegate TJ, Dibner J, Kitchell ML, Uni Z, Lilburn MS. Effect of turkey (Meleagridis gallopavo) breeder hen age and egg size on poult development. 2. Intestinal villus growth, enterocyte migration, and proliferation of the turkey poult. Comparative Biochemistry and Physiology B 1999;124:381-389.

Baranyiova E. Influence of deutectomy, food intake and fasting on the digestive tract dimensions in chickens after hatching. Acta Veterinaria Brno 1972;41:373-374

Baranyiova E, Holman J. Morphological Changes in the Intestinal Wall in Fed and Fasted Chickens in the First Week after Hatching. Acta Veterinaria Brno 1976;45:151-158.

Batal $A B$, Parsons CM. Effect of fasting versus feeding oasis after hatching on nutrient utilization in chick. Poultry Science 2002;81:853-859.

Bigot K, Mignon-Grasteau S, Picard M, Tesseroud S. Metabolism and nutrition: effects of delayed feed intake on body, intestine and muscle development in neonate broilers. Poultry Science 2003;82:781-788.

Dibner JJ. Early feeding and development of the immune system in neonatal poultry. Journal of Applied Poultry Research 1998;7:425- 436

Ganjali H, Ahmad RR, Heydar Z. Effect of post hatch delayed access to feed on performance, GIT physical and histological development yolk absorption in young broiler chicks. Biomedical and Pharmacology Journal 2015;8(2):945-955.

Geyra A, Uni Z, Sklan D. The effect of fasting at different ages on growth and tissue dynamics in the small intestine of the young chick. British Journal of Nutrition 2001a;86:53- 61

Geyra A, Uni Z, Sklan D. Enterocyte dynamics and mucosal development in the posthatch chick. Poultry Science 2001 b;80:776-782.

Gonzales E, Kondo N, Saldanha ESPB, Loddy M, Careghi M, Ducuypere E. Performance and physiological parameters of broiler chickens subjected to fasting on the neonatal period. Poultry Science 2003;82:1250-1256. 
Govaerts T, Room G, Buyse J, Lippens M, De Groote G, Decuypere E. Early and temporary quantitative food restriction of broiler chickens. 2. Effects on allometric growth and growth hormone secretion. British Poultry Science 2000;41(3):355-362.

Iji P, Saki A, Tivey ADR. Body and intestinal growth of broiler chicks on a commercial starter diet. 1. Intestinal weight and mucosal development. British Poultry Science 2001a;42:505-513.

Imondi AR, Bird F. The sites of nitrogen absorption from the alimentary tract of the chicken. Poultry Science 1965;44(4):916-920.

Imondi AR, Bird H. The turnover of intestinal epithelium in the chick. Poultry Science 1996;45:142-147.

Isshiki Y. Feed digestibility in different intestinal parts of chickens. Animal Science Journal 1989;60:1082-1092.

Lallo $\mathrm{CHO}$, Ramraj S. The performance of improved pekin and mule duck strains reared under intensive conditions in a humid tropical environment. Proceedings of the International Congress on Tropical Agriculture; 2008 Nov 30 - Dec 5.; Trinidad: Faculty of Science \& Agriculture; 2008. p.309-317

Loudon AH, Shanmugasundaram R, Lilburn MS, Selvaraj RK. Intestinal physiology and regulatory T-cell response to immediate or delayed access to feed and water in Pekin ducklings. Poultry Science 2011; 90:2041-2046

Maiorka A, Satin E, Dahlke F, Boleli IC, Furlan RL, Macari M. Posthatching water and feed deprivation affect the gatrointestinal tract and intestinal mucosa development of Boiler Chicks. Journal ofApplied Poultry Research 2003;12:483- 492 .

Moran ET. Effects of egg weight, glucose administration at hatch, and delayed access to feed and water on poultry at 2 weeks of age. Poultry Science 1990;69:1718-1723

Moran ET, Reinhart BS. Poultry yolk sac amount and composition upon placement effect of breeder age, egg mass, sex and subsequent change with feeding and fasting. Poultry Science 1980;59:1521-1528.

Murakami H, Akiba Y, Horiguchi M. Growth and utilization of nutrients in newly hatched chicks with or without removal of residual yolk. Growth, Development and Aging 1992;56:75-84.

Nistan Z, Ben- Auraham G, Zoref Z, Nir I. Growth and development of the digestive organs and some enzymes in broiler chicks after hatching. British Poultry Science 1991a;32:515-523.

Noy Y, Sklan D. Post-hatch development in poultry. Journal of Applied Poultry Research 1997;6:344-354.

Noy Y, Sklan D. Yolk utilization in the newly hatched poultry. British Poultry Science 1998;39:446-451.

Noy Y, Sklan D. Energy utilization in a newly hatched chick. Poultry Science 1999b;78:1750-1756.

NRC- National Research Council. Nutrient requirements of poultry. $9^{\text {th }}$ rev. ed. Washington: National Academy Press; 1994

Przystalski A. Changes in structure and size of alimentary canal during postembryonal development of the tree sparrow, Passer m. montanus L. Acta Biol Cracoy. 1987a;24:93-105.

Raheja KL, Tepperman J, Tepperman HM. The Effect of age on intestinal glucose transport in the chick (Gallus domesticus). Comparative Biochemistry and Physiology A 1977;58:245-248.

Ravindran V. Development of digestive function in neonatal poultry:physiological limitation and potential. Proceedings from Australia Poultry Science Symposium 2003;52:423-429.
Saki AA. Effect of post-hatch feeding on broiler performance. Internatioonal Journal of Poultry Science 2005;4(1):4-6.

Sell JL, Angel CR, Piquer FJ, Mallarino EG, Albatshan HA. Developmental patterns of selected characteristics of the gastrointestinal-tract of young turkeys. Poultry Science 1991;70:1200-1205.

Sklan D. Hydrolysis and absorption in the small intestines of post-hatch chicks. Poultry Science 2000;79:1306-1310.

Sklan D, Noy Y, Hoyzman A. Rozenboim I. Decreasing weight loss in the hatchery by feeding chicks and poults in hatching trays. Journal of Applied Poultry Research 2000;9:142-148.

Sklan D. Development of the digestive tract of poultry. World's Poultry Science Journal 2001;57:415-428.

Sklan D. Early nutrition and its effect on lifelong productivity in poultry. Recent Advances in Animal Nutrition in Australia 2003;14:75-79.

Sulistiyanto B, Akiba Y, Sato K. Energy utilization of carbohydrate, fat and protein sources in newly hatched broiler chicks. British Poultry Science 1999;40:653-659

Suvarna SR. Ontogeny of glucose transport in turkey intestines [dissertation]. Raleigh (NC): North Carolina State University; 1999.

Tabeidan SA, Samie A, Pourreza J, Sadeghi GH. Effect of fasting or post hatch feed type on intestinal morphology in broilers. Proceedings of the 3rd International Conference on Life Science and Technology; 2011; Singapore: IACSIT Press; 2001. p.69-74.

Uni Z, Ganot S, Sklan D. Post hatch development of mucosal function in broiler small intestine. Poultry Science 1998;77:75-82.

Uni Z, Noy $Y$, Sklan D. Development of the small intestine in heavy and light strain chicks before and after hatching. British Poultry Science 1995b;36:64-71.

Uni Z, Noy Y, Sklan D. Posthatch development of small intestinal function in the poult. Poultry Science 1999;78:215-222.

Uni Z, Smirnov A, Sklan D. Pre- and post-hatch development of goblet cells in the broiler small intestine: effect of delayed access to feed. Poultry Science 2003;82:320-327.

Wang L, Li J, Chen YM, Duan XL. Morphological change in rat jejunal mucosal epithelia and cell proliferation and apoptosis in different months. Acta Zoologica Sinica 2003;49:91-97.

Wijtten $\mathrm{P}$, Langhout $\mathrm{D}$, Verstegen $\mathrm{M}$. Small intestine development in chicks after hatch and in pigs around the time of weaning and its relation with nutrition:A review. Acta Agriculturae Scandinavica, Section A Animal Science 2012;62(1):1-12.

Yamauchi K, Kamisoyama H, Isshiki Y. Effects of fasting and re-feeding on structures of the intestinal villi and epithelial cells in White Leghorn hens. British Poultry Science 1996;37:909-921.

Yang HM, Wang SR, Chen WL, Zhou OY, Fan L, Xu MJ. Effect of early feeding on the yolk nutrient utilization by goslings after hatch. Archiv für Geflügelkunde 2008;72(6):264-268.

Yegani M, Korver DR. Factor affecting intestinal health in poultry. Poultry Science 2008;87:2052-2063

Zarghi $\mathrm{H}$. Influence of delayed access to feed on gastrointestinal tract development in Japenese quail (Coturnix Japonica). International Conference on Modern Research in Agricultural Science and Environment; 2005; Kualalumpur. p.2-9. 